\title{
Disturbances of the Gut Microbiota, Sleep Architecture, and mTOR Signaling Pathway in Patients with Severe Obstructive Sleep Apnea-Associated Hypertension
}

\author{
Chih-Yuan Ko $\mathbb{D},{ }^{1,2,3,4}$ Huan-Zhang Su, ${ }^{1,4,5}$ Li Zhang, ${ }^{1,4}$ and Yi-Ming Zeng $\mathbb{D}{ }^{1,4}$ \\ ${ }^{1}$ Department of Respiratory and Critical Care Medicine, The Second Affiliated Hospital of Fujian Medical University, \\ Quanzhou 362000, China \\ ${ }^{2}$ Department of Clinical Nutrition, The Second Affiliated Hospital of Fujian Medical University, Quanzhou 362000, China \\ ${ }^{3}$ School of Public Health, Fujian Medical University, Fuzhou 350122, Fujian, China \\ ${ }^{4}$ Respiratory Medicine Center of Fujian Province, Quanzhou 362000, China \\ ${ }^{5}$ Department of Respiratory, The First Hospital of Quanzhou, Quanzhou 362000, China
}

Correspondence should be addressed to Chih-Yuan Ko; yuanmomoko@gmail.com and Yi-Ming Zeng; zeng_yi_ming@126.com

Received 23 September 2021; Revised 9 November 2021; Accepted 16 November 2021; Published 30 November 2021

Academic Editor: Kwok Leung Ong

Copyright $(92021$ Chih-Yuan Ko et al. This is an open access article distributed under the Creative Commons Attribution License, which permits unrestricted use, distribution, and reproduction in any medium, provided the original work is properly cited.

Intermittent hypoxia and sleep fragmentation are pathophysiological processes involved in obstructive sleep apnea (OSA) which affect gut microbiota, sleep architecture, and mTOR signaling pathway. However, the involvement of these elements in the pathogenesis mechanism of OSA-associated hypertension remains unclear. Therefore, this study investigated whether the OSAassociated hypertension mechanism is regulated by the gut microbiota and mTOR signaling pathway. Patients were diagnosed by polysomnography; their fecal samples were obtained and analyzed for their microbiome composition by 16S ribosomal RNA pyrosequencing and bioinformatics analysis. Transcript genes on fasting peripheral blood mononuclear cells (PBMCs) were examined using Illumina RNA-sequencing analysis. Totally, we enrolled 60 patients with severe OSA [without hypertension $(n=27)$ and with hypertension $(n=33)]$ and 12 controls (neither OSA nor hypertension). Results revealed that severe-OSA patients with hypertension had an altered gut microbiome, decreased short-chain fatty acid-producing bacteria $(P<0.05)$, and reduced arginine and proline metabolism pathways $(P=0.001)$, compared with controls; also, they had increased stage N1 sleep and reduced stages N2 and N3 sleep accompanied by repeated arousals $(P<0.05)$. Analysis of PBMCs using the Kyoto Encyclopedia of Genes and Genomes database showed that the mTOR signaling pathway $(P=0.006)$ was the most important differential gene-enriched pathway in severe-OSA patients with hypertension. Our findings extend prior work and suggest a possibility that the regulation of the mTOR signaling pathway is involved in developing OSA-associated hypertension through its interaction with the disturbance of the gut microbiome and sleep architecture.

\section{Introduction}

Obstructive sleep apnea (OSA) is a common clinical sleep disorder affecting the entire body, and it is related to the increased morbidity and mortality of cardiovascular and cerebrovascular diseases [1]. Intermittent hypoxia (IH) and sleep fragmentation (SF) are important hallmarks of OSA which are partially attributed to decreased airflow (hypopnea) or interrupted breathing (apnea), accompanied by decreased blood oxygen saturation and hypercapnia. These pathophysiological processes can lead to a series of complications, including hypertension, cerebrovascular events, or metabolic disorders [2].

OSA is an independent risk factor for hypertension. The severity of hypertension positively correlates with the apneahypopnea index (AHI). Continuous positive airway pressure (CPAP) treatment can substantially reduce the blood pressure of patients with OSA and hypertension (OSAHTN), especially those with refractory hypertension [3, 4]. $\mathrm{IH}$ can lead to decreased oxygen saturation, increased 
vascular pressure, excessive activation of sympathetic nerve activity, autonomic dysfunction, and apnea that awakens the central nervous system. It can ultimately lead to vascular endothelial dysfunction and multiple organ damage. Its underlying mechanism involves a cascade of inflammation and oxidative stress [5]. CPAP treatment has a protective effect on the neurocognition of patients with OSA whether gut microbiota is involved in the neurocognitive physiological mechanism of OSA unclearly [6]. Recent research has shown that gut microbiota plays a critical role in regulating the risk of chronic diseases and maintaining intestinal immunity and systemic homeostasis. They significantly affect the onset of obesity, cardiometabolic abnormalities, and mental illness [7]. Interestingly, these lesions can be seen in patients with OSA.

SF can cause vital organ disruptions independent of IH. $\mathrm{SF}$ is with repeated arousals implicated in overactivation of sympathetic neural activity. The principal mechanism of OSA-induced brain damage involves disrupting different stages of sleep by causing rapid eye movement (REM) and non-REM (NREM) sleep interruptions [8]. Studies have found that sleep architecture changes and reduced deep sleep time may lead to increased blood pressure, indicating that SF may have a certain mechanism in OSA-associated hypertension [9-11]. Moreover, SF promotes obesity and metabolic abnormalities, mediated by changes in the host intestinal microbiota and systemic adipose tissue inflammation accompanied by insulin resistance $[12,13]$.

Animal models mimicking IH and SF in OSA have demonstrated that the gut microbiota and their metabolites, such as bile acids and short-chain fatty acid (SCFAs), were altered $[12,14-16]$. Our previous human study revealed that the gut microbiota is unbalanced. The microbiome's metabolic function analysis shows changes also combined with elevated proinflammatory cytokines in patients with different OSA severity levels [17]. Additionally, we found that the sleep architecture is disrupted, especially in patients with an AHI $\geq 15$ who have the Prevotella enterotype [18]. The gut microbiota may be involved in regulating the structure of sleep and blood pressure through the brain-gut axis [19]. Therefore, the correlations between the gut microbiome's role in the development of OSA itself and its complications are worth investigating.

However, the mechanism of OSA-associated hypertension remains unclear. Animal experiments with rodents have confirmed that the gut microbiotal composition can be altered by regulating the mechanistic target of rapamycin (mTOR) signaling pathway under such conditions as hypoxia and energy deficiency $[20,21]$. Furthermore, the correlation between gut microbiome alteration and blood pressure affects the development of hypertension [22, 23]. mTOR is a highly conserved serine/threonine-protein kinase whose signaling pathway is involved in the regulation of oxidative stress, immunity, metabolic disorders, and inflammation [24-28]. Moreover, probiotics regulating immune balance via the mTOR signaling pathway are effective in treating hypertension [23].

We hypothesized that the changes in the gut microbiota and mTOR signaling pathway in patients with OSA are involved in developing OSA-associated hypertension. Thus, we investigated whether the gut microbiota regulates the mechanism of OSA-associated hypertension, sleep architecture, and mTOR signaling pathway by evaluating the gut microbiota, polysomnography (PSG) data, and mTOR levels of peripheral blood mononuclear cells (PBMCs) of severeOSA patients with hypertension and those without hypertension.

\section{Materials and Methods}

2.1. Subjects. The Institutional Review Board of the Second Affiliated Hospital of Fujian Medical University (Quanzhou, China) approved this study (approval no. 2017-78). Patients with unexplained diarrhea, infection, or gastrointestinal diseases and who used antibiotics or probiotics approximately 1 month before recruitment began were excluded. In total, 72 subjects were recruited. They underwent a full night of PSG (SOMNOscreen plus; SOMNOmedics, Randersacker, Germany) conducted by technologists in a sleep laboratory. Fasting blood or fecal samples were collected on the following morning.

2.2. OSA Assessments. OSA was evaluated as previously described [29]. All the patients underwent PSG with a computerized polysomnographic system, which covered electro-oculography, electroencephalography, electromyography, electrocardiography, and dynamic blood pressure measurements, including systolic blood pressure (SBP) and diastolic blood pressure (DBP). High blood pressure was defined as SBP $\geq 140 \mathrm{mmHg}$ and/or DBP $\geq 90 \mathrm{mmHg}$ or determined based on the patients' self-reported use of medications for hypertension. After the examination, AHI obtained the total number of apnea episodes (continuous cessation of airflow for at least $10 \mathrm{~s}$ ) and hypopnea episodes (a 30\% drop in the nasal pressure excursion for $\geq 10$ seconds with $\geq 3 \%$ oxygen desaturation or arousal) by dividing the total sleep time to calculate the number of hypopneas plus apneas per hour during sleep, according to the diagnostic criteria of the 2012 American Academy of Sleep Medicine. Patients with an AHI $\leq 5$ were assigned to the non-OSA control group, whereas patients with an AHI $\geq 30$ were classified as the severe-OSA group.

2.3. Sampling, DNA Extraction, and $16 S$ rRNA Gene Amplification Sequencing, Bioinformatic, Predictive Function. All fresh fecal samples were collected and stored in a Microbiome Test Kit (G-BIO Biotech, Hangzhou, China). 16S ribosomal RNA sequencing was conducted as described in our previous study [17]. The total sequence data of the fecal samples were analyzed for microbiome taxa based on the Quantitative Insights into Microbial Ecology bioinformatics pipeline for performing taxonomy assignments using the operational taxonomic unit method. The Phylogenetic Investigation of Communities by Reconstruction of Unobserved States bioinformatics software package and Kyoto Encyclopedia of Genes and Genomes (KEGG) database were used to predict bacterial metabolic functions. 
2.4. Establishment of the Individuals' Transcriptome of PBMCs for RNA Sequencing. In total, $5 \mathrm{~mL}$ of venous blood was collected from 6 non-OSA subjects with an AHI $\leq 5$ without hypertension and 6 OSA-HTN patients with an AHI $\geq 30$. The complementary DNA was reverse-transcribed from the total RNA extracted from PBMCs and prepared according to reported protocols [30]. According to the manufacturer's instructions, RNA quality was analyzed using the Agilent Bioanalyzer system (Agilent, USA). RNA-sequencing assay was carried out on the Illumina HiSeq 2000 platform (Illumina, San Diego, CA, USA), and 150 base pairs of paired-end reads were obtained. A complementary DNA library was established, and sequencing was performed according to the Illumina standard protocol published by Novogene (https://en.novogene.com).

The FastQC software was used for raw read count data from the quality control of RNA-sequencing libraries. Clean reads were obtained by evaluating the $\mathrm{QC}$ and $\mathrm{A} / \mathrm{T} / \mathrm{G} / \mathrm{C}$ content and filtering the raw reads. The specific criteria were as follows: (1) removal of reads with a connector (adapter); (2) removal of reads with $\mathrm{N}$ with a percentage greater than $10 \%$; (3) removal of low-quality reads (reads in which the number of bases with quality value Qphred $\leq 20$ accounts for more than $50 \%$ of the whole read). All downstream analyses were based on the clean data with high quality. Bioinformatics software analysis was performed with the $\mathrm{R}$ package DESeq using sequencing results for reference sequence comparison, expression statistics, and differential gene screening. Genes with a $Q$ value of $<0.05$ were considered differentially expressed and retained for further analysis. Cluster analysis of differentially expressed genes was applied to describe their expression patterns under different treatments. KEGG enrichment analysis of the biological processes and molecular functions of up- and downregulated differentially expressed genes between control patients without OSA and patients with OSA-HTN was performed via Enrichr. The $p$-value of enrichment analysis has been adjusted in false discovery rate and was computed using the Fisher exact test.

2.5. Statistical Analyses. Differences in fecal microbiota were analyzed and performed by the Kruskal-Wallis test and R statistics, as appropriate. Further data analysis by $t$-test or one-way analysis of variance (ANOVA) was undertaken with SPSS version 19.0 (SPSS Inc., Chicago, IL, USA), and the data are expressed as the mean \pm standard deviation (SD). Significant differences within groups were analyzed with ANOVA, followed by post hoc Fisher's least significant difference corrections for multiple normally distributed variance data comparisons. We considered a two-sided $P<0.05$ to be statistically significant.

\section{Results}

3.1. Participant Characteristics. After PSG assessment, we recruited a total of 60 patients with severe OSA (without hypertension, $n=27$; with hypertension, $n=33$ ) and 12 nonOSA without hypertension control subjects (AHI of $\leq 5$ ).
Weight $(P<0.001)$, body mass index $(P<0.001)$, waist circumference $(P<0.001)$, and hip circumference $(P=0.002)$ in the OSA group were significantly greater than those in the non-OSA group (Table 1).

3.2. Alterations in Taxa among Groups. We examined the mean community diversity indices (Supplementary Figure 1S). At the phylum level, we found no significant differences in the $\mathrm{F} / \mathrm{B}$ ratio (Figure $1(\mathrm{a})$ ). The relative abundance of Megamonas $(P=0.034), \quad$ Gemmiger $(P=0.012)$, Dialister $(P=0.002)$, and Oscillibacter $(P=$ $0.006)$ genera in the non-OSA group was significantly higher than in the OSA group. The relative abundance of Parabacteroides $(P=0.027)$, Gemmiger $(P=0.026)$, Dialister $(P=0.008), \quad$ Oscillibacter $\quad(P=0.019), \quad$ Akkermansia $(P=0.041)$, and Odoribacter $(P=0.019)$ genera in the nonOSA group was significantly higher than those in the OSAHTN group. Clostridium XIVa $(P=0.046)$ was in the OSA group significantly higher than in the OSA-HTN group. Conversely, $\mathrm{f}$ _Prevotellaceae $(P=0.030)$ and Bifidobacterium $(P=0.021)$ genera were in the OSA group significantly lower than in the OSA-HTN group (Figure 1(b)).

3.3. Predictive Functional Analysis. Based on the KEGG database, significant differences in the fecal microbiome between the study groups were detected. Pathways of base excision repair $(P=0.027)$ as well as tropane, piperidine, and pyridine alkaloid biosynthesis $(P=0.041)$ were significantly higher than in the OSA group. Pathways of arginine and proline metabolism $(P=0.001)$, pentose and glucuronate interconversions $(P=0.048)$, base excision repair $(P=0.026)$, the Vibrio cholerae pathogenic cycle $(P=0.018)$, bisphenol degradation $(P=0.040)$, linoleic acid metabolism $(P=0.036)$, and flavonoid biosynthesis $(P=0.025)$, as well as flavone and flavonol biosynthesis $(P=0.045)$, in the non-OSA group were significantly higher than those in the OSA-HTN group (Figure 1(c)).

3.4. PSG Parameter Analysis. N1 sleep stage, arousal index in NREM, total sleep arousal index, apnea-hypopnea index, obstructive apnea index, central apnea index, mixed apnea index, hypopnea index, oxygen desaturation index, blood pressure elevation index, and the highest systolic blood pressure in the non-OSA group were significantly lower than the OSA group and the OSA-HTN group (Table 2). On the contrary, N2 sleep stage, N3 sleep stage, lowest oxygen saturation, and average oxygen saturation in the non-OSA group were significantly higher than the OSA group and the OSA-HTN group (Table 2). The OSA-HTN group had the highest average systolic blood pressure and average diastolic blood pressure among three groups (Table 2).

3.5. RNA-Sequencing Analysis. The differentially expressed mRNAs between the OSA-HTN group and the non-OSA group that were previously selected were further screened by heat maps using unsupervised hierarchical cluster analysis (Figure 2(a)). Compared with those of the non-OSA group, 
TABle 1: Participant characteristics.

\begin{tabular}{lccc}
\hline & $\begin{array}{c}\text { Non-OSA } \\
(n=12)\end{array}$ & $\begin{array}{c}\text { OSA } \\
(n=60)\end{array}$ & $P$ value \\
\hline Gender (male/female) & $6 / 6$ & $57 / 3$ & $\mathrm{NA}^{\mathrm{a}}$ \\
Age (years, mean $\pm \mathrm{SD})$ & $38.25 \pm 8.06$ & $42.12 \pm 7.63$ & 0.117 \\
Height $(\mathrm{cm})$ & $165.25 \pm 8.41$ & $168.28 \pm 6.81$ & 0.180 \\
Weight $(\mathrm{kg})$ & $65.38 \pm 8.96$ & $81.11 \pm 12.01$ & $<\mathbf{0 . 0 0 1}$ \\
Body mass index $\left(\mathrm{kg} \mathrm{m}^{-2}\right)$ & $23.81 \pm 2.59$ & $28.49 \pm 3.76$ & $<\mathbf{0 . 0 0 1}$ \\
Waist circumference $(\mathrm{cm})$ & $80.00 \pm 6.73$ & $97.32 \pm 8.04$ & $<\mathbf{0 . 0 0 1}$ \\
Hip circumference $(\mathrm{cm})$ & $94.42 \pm 7.03$ & $100.89 \pm 6.09$ & $\mathbf{0 . 0 0 2}$ \\
\hline
\end{tabular}

${ }^{a}$ N/A: not analyzed. Non-OSA: subjects with apnea-hypopnea index (AHI) $\leq 5$ without hypertension; OSA: patients with severe OSA (AHI $\geq 30)$ with/ without hypertension. The statistical analysis was performed with a $t$-test. $P$ values $<0.05$ were considered significant.

the PBMC genes of the OSA-HTN group differed in that 53 genes showed upregulation, and 292 genes exhibited downregulation (Figure 2(b)). The top 10 differentially expressed mRNAs are listed in Table 3.

The results of the KEGG pathway analysis showed that the mTOR signaling pathway was enriched with the largest number of differential genes $(P=0.006)$; the $Q$ value was $<0.05$ (Figure 2(c)). We only listed the top 5 up- or downregulated genes of the relevant pathways, with the number of genes showing their corresponding gene IDs. On the other hand, in the mTOR signaling pathway, the WD repeat domain 24; disheveled segment polarity protein 1; HRas proto-oncogene, GTPase; Unc-51 like autophagy activating kinase 1; serine/threonine kinase 11 (STK11); and telomere maintenance 2 genes were downregulated in the OSA-HTN group, but not in the non-OSA group (Table 4).

\section{Discussion}

In this study, we found that the severe-OSA patients with hypertension and those without hypertension had obvious sleep architecture disturbances that manifested as increased stage N1 sleep as well as decreased stages N2 and N3 sleep accompanied by repeated arousals. Their gut microbiota's relative abundance decreased, especially SCFA-producing bacteria involving Megamonas, Gemmiger, Dialister, or Oscillibacter. The downregulation of arginine and proline metabolism was examined by functional analysis of microbiota. In addition, the mTOR signaling pathway was enriched with the largest number of differential genes from PBMCs.

4.1. OSA-Induced Gut Microbial Dysbiosis. OSA is a common sleep disorder that is characterized by repeated upper airway obstruction causing IH and SF. In patients with OSA, arousal is an important mechanism for reopening the upper airway and plays a crucial role in maintaining the upper airway in an open state when apnea occurs [2]. Moreover, it is an important factor in disruptions in the sleep architecture and gut microbiome $[8,14]$. Because of the repeated hypoxia/reoxygenation periodic changes in the intestine, the appearance of a hypoxic environment in the intestine is conducive to anaerobic bacterial growth. An increase in the number of anaerobic bacteria can reduce the integrity of intestinal epithelial cell tight junctions by increasing intestinal mucosal permeability and bacterial translocation, which contribute to gut microbiota dysbiosis [12-14].

The intestine is the human body's largest mucosal immune defense line. Intestinal symbiotic microorganisms can have a role in host immune regulation, whereas SCFAs can regulate immune cells and play an important role in maintaining the balance of the intestinal immune microenvironment [31]. In this study, we found that the abundance of SCFA-producing bacteria decreased in the severe-OSA patients with hypertension and those without hypertension. SCFAs have a direct anti-inflammatory effect on the intestine, promote mucus synthesis, reduce bacterial translocation, maintain intestinal integrity, and reduce the host's intestinal inflammatory response [31]. Our previous study also observed a decrease in SCFAproducing bacteria and increased proinflammatory $c y-$ tokines in OSA patients [17]. Increased levels of inflammatory factors in OSA are deemed to be potentially related to its pathophysiological processes and complications [5]. SCFAs can reduce the release of tumor necrosis factor (TNF)- $\alpha$, interleukin (IL)-2, and IL-6 through the histone deacetylase inhibitory pathway via the G-protein-coupled receptor (GPR) 41 and GPR43 receptors [31]. These data suggest that the changes in SCFA-producing bacteria and cytokines in OSA may be related to its pathophysiological processes.

\subsection{OSA-Induced Disruption of Sleep Architecture Impact on} the Gut Microbiota and Metabolic Changes. Interestingly, studies have shown that changes in sleep rhythm can be attributed to changes in the intestinal microbiota and the body's metabolism. Metabolic changes induced by SF may be partly mediated by gut microbiotal taxa alteration $[12,32]$. The structural components and metabolites of intestinal bacteria, such as bacterial peptides and lipopolysaccharides, can induce IL- $1 \beta$ or TNF- $\alpha$ production, which in turn can induce the NREM stage; on the other hand, cortisol can inhibit the synthesis of these cytokines, which have the same rhythm as cortisol [33]. Therefore, we hypothesized that OSA may affect the gut microbiome owing to changes in the sleep structure and that dysbiosis in the gut microbiota may also regulate the sleep structure through their metabolites. The gut microbiotal composition and activity are related to the development and function of the central nervous system. The bidirectional network between the brain and the intestine is the braingut axis. The gut microbiota are related to the hypothalamic-pituitary-adrenal axis; both systems affect the normal sleep architecture $[18,19,34]$.

A reduction in deep sleep may be related to increased blood pressure and may increase refractory hypertension prevalence [9]. A study has shown that post-REM sleep deprivation led to vascular endothelial dysfunction and 


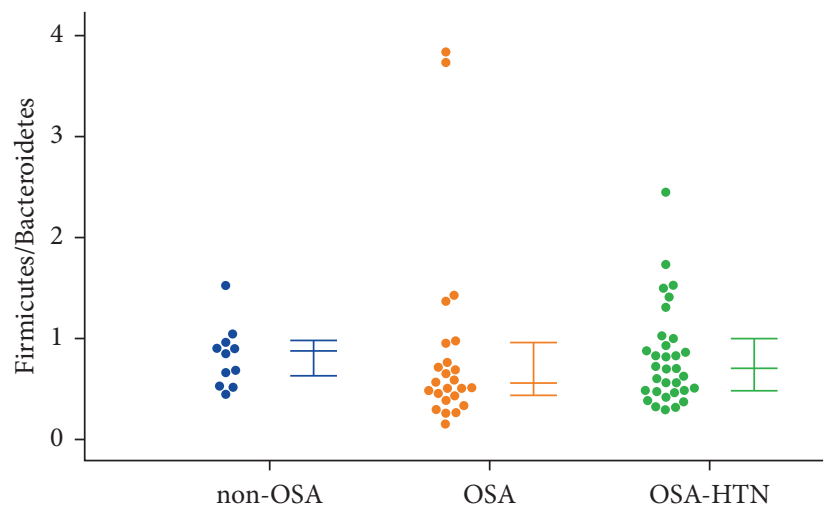

(a)

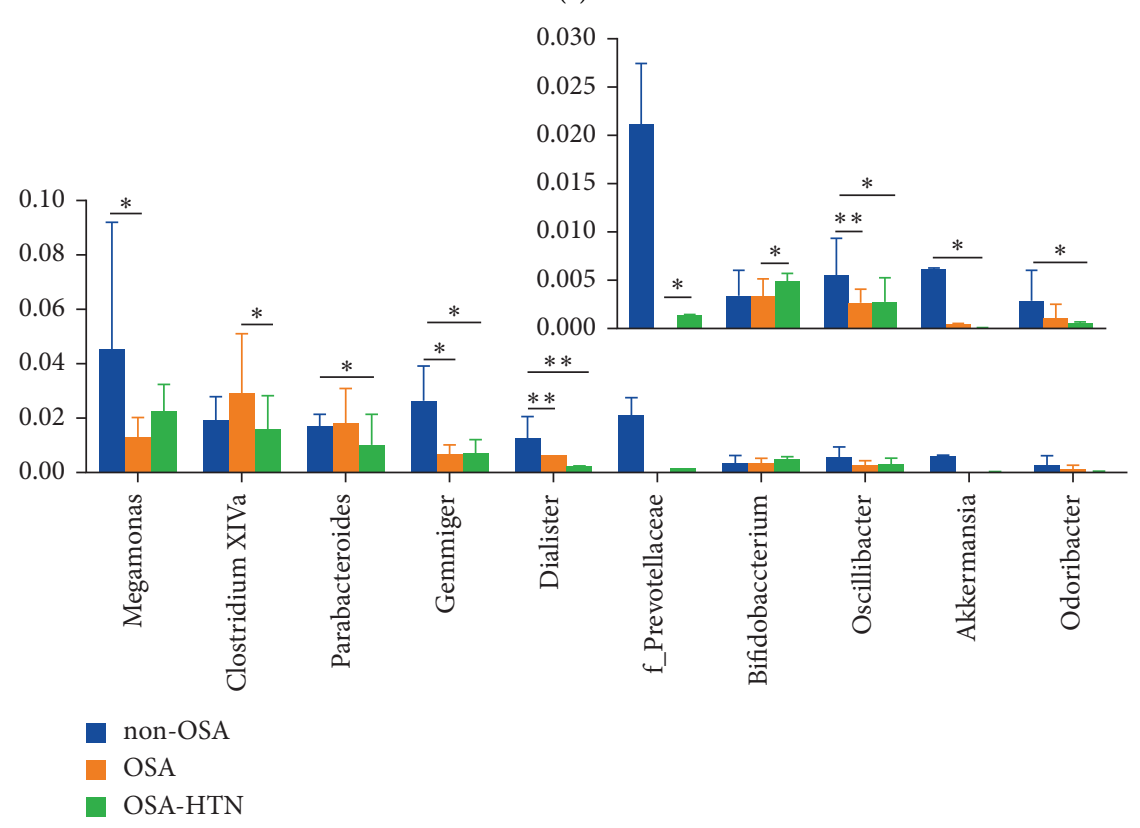

(b)

FIgURE 1: Continued. 


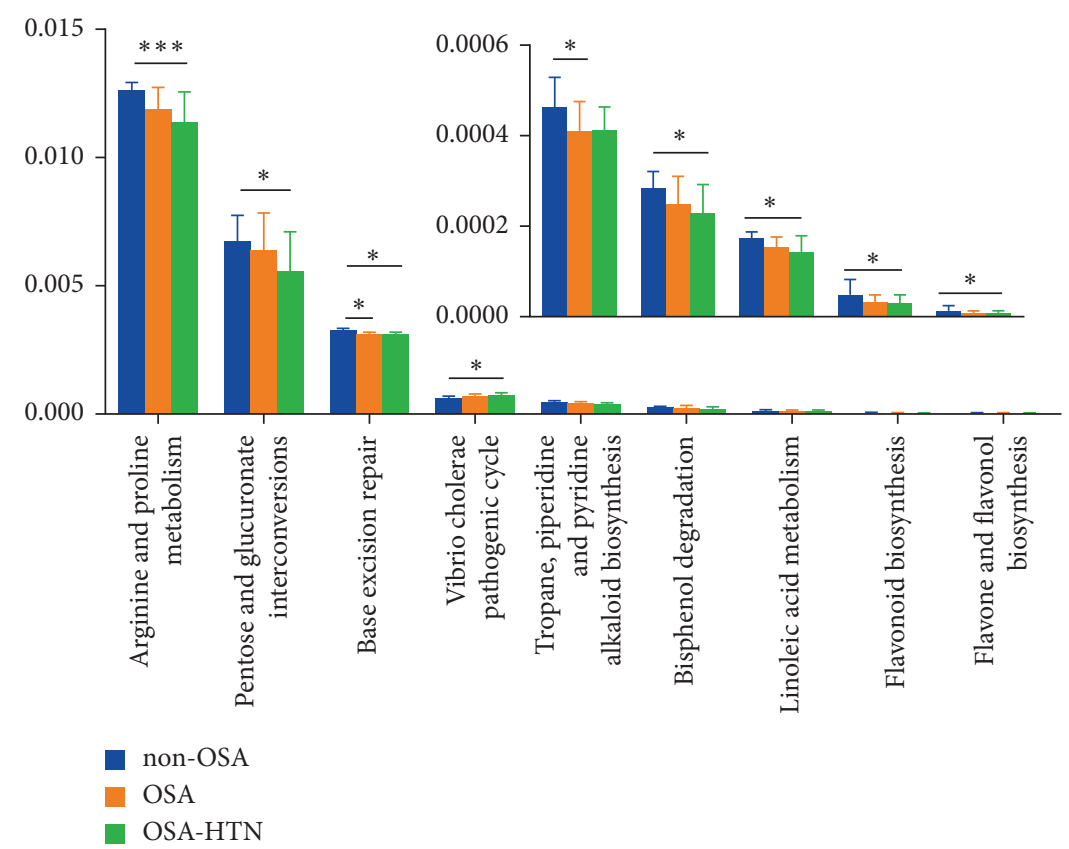

(c)

FIgURE 1: Alteration of the gut microbiome in severe obstructive sleep apnea (OSA) patients with hypertension and those without hypertension. (a) The Firmicutes to Bacteroidetes ratio is similar across groups at the phylum level. (b) The Kruskal-Wallis test was used to analyze differences in the relative abundance of the fecal microbiota at the genera level. (c) Predictive functional analysis revealed that the fecal microbiome exhibited significant Kyoto Encyclopedia of Genes and Genomes pathways. Statistical analysis was performed with the Kruskal-Wallis test. Non-OSA: apnea-hypopnea index (AHI) $\leq 5$ without hypertension, OSA: severe OSA (AHI $\geq 30$ ) without hypertension, and OSA-HTN: severe OSA with hypertension. ${ }^{*} P<0.05,{ }^{* *} P<0.01$, and ${ }^{* * *} P<0.001$ compared with the non-OSA group or the OSA group.

increased blood pressure in rats [10]. The arousal index is negatively correlated with the N3 stage, and sleep architecture disruptions in OSA patients may be related to frequent awakenings at night-that is, repeated awakenings lead to SF, increased light sleep times, and reduced deep sleep and REM sleep times. Reduced sleep in the N3 stage is considered to be an independent risk factor for hypertension as the risk of hypertension increases in patients with lowlevel stage N3 sleep [11]. Therefore, we infer that OSA manifests as frequent arousals, leading to SF and decreased deep sleep, which may play an important role in OSA-associated hypertension. However, the sleep architecture of the patients with OSA-HTN was similar to that of the patients with OSA only. There are little researches on sleep architecture disturbances of OSA patients with or without hypertension. Stages N2 sleep and arousal times were increased of OSA patients with hypertension than patients with hypertension [35]. Recently, Gürün Kaya et al. [36] found that only the arousal index was significantly higher in severeOSA patients with hypertension than severe-OSA patients; there was no statistically significant difference in stages $\mathrm{N} 1$, $\mathrm{N} 2$, and N3 sleep.

4.3. The Effect of Metabolites of Gut Microbiota on Blood Pressure. Notably, functional analysis of the gut microbiota revealed that arginine metabolism in patients with OSAHTN was significantly reduced. Arginine participates in the synthesis of nitric oxide (NO), which declines with increased blood pressure. Sleep deprivation during REM reduces the phosphorylation levels of endothelial NO synthase, NO, and cyclic guanosine monophosphate in the aorta, which impairs NO-mediated vasodilation as well as vasomotor dysfunction and affects the development of hypertension [10]. Moreover, a reduction in SCFA-producing bacteria is another cause of OSA-associated hypertension. The interaction between SCFAs and the GPR olfactory receptor 78 is believed to be involved in regulating blood pressure. SCFAs bind to olfactory receptor 78 on the renal vascular smooth muscle to regulate renin's release, which leads to blood pressure alteration [37].

4.4. OSA Alters Microbiome, Sleep Architecture, and mTOR Signaling Pathway to Develop Hypertension. Moreover, sleep deprivation attenuates the mTOR signaling pathway's expression, which can regulate central and peripheral circadian clock functions $[27,28]$. mTOR may be linked to the intestinal microbiota. The relative abundance of such microbiota as Oscillibacter and Ruminococcus in mice fed with a high-fat diet was altered using resveratrol and rapamycin to inhibit the mTOR pathway [26]. Through changes in phosphatidylinositol 3-kinase/phosphorylated protein kinase $\mathrm{B}$, the mTOR pathway can be adjusted to regulate the intestinal barrier function directly, thereby altering the gut microbiotal composition [20]. Inhibition of 
TABLE 2: Polysomnographic data analysis among non-OSA, OSA, and OSA-HTN.

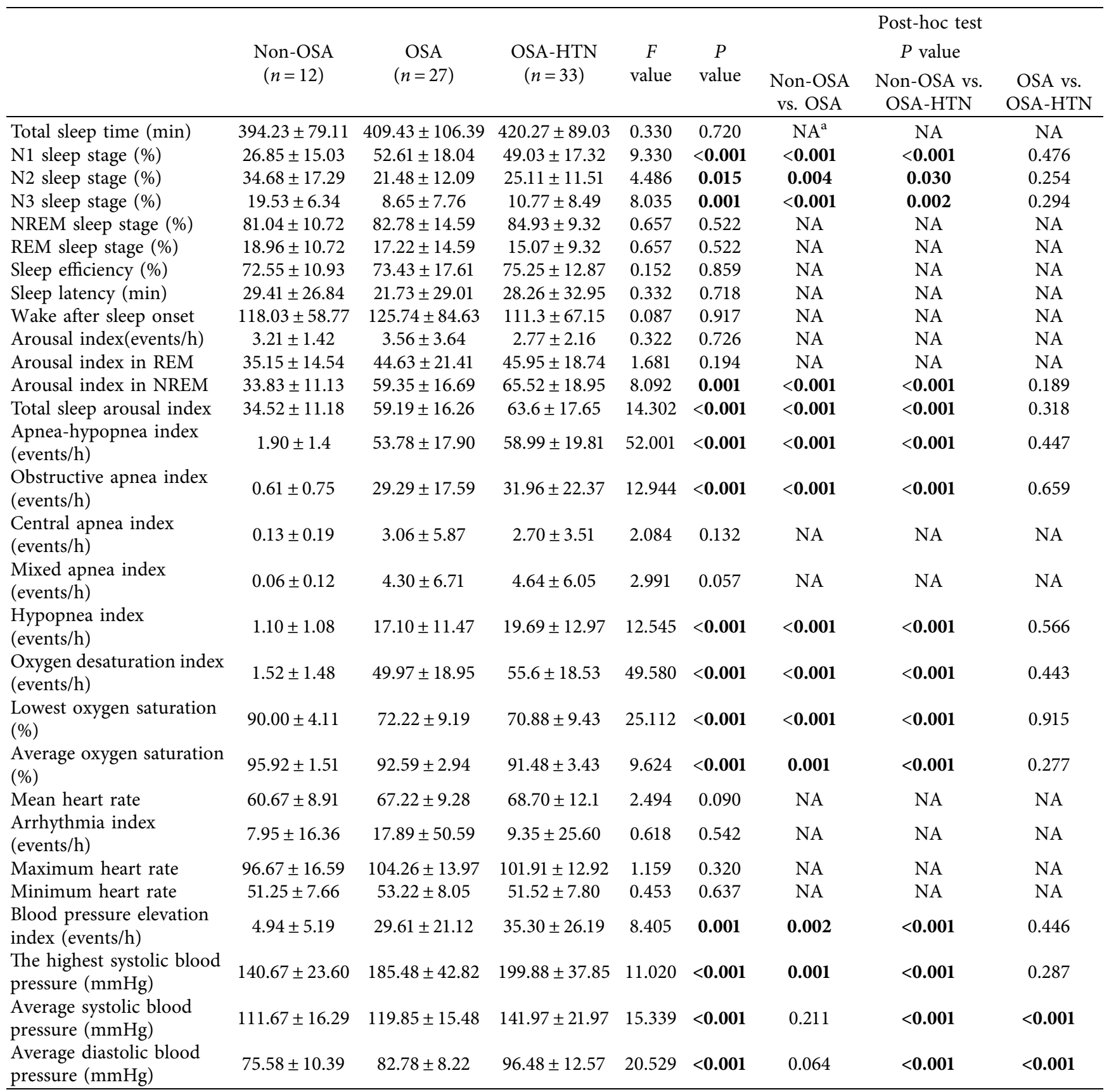

${ }^{a}$ Not analyzed. Non-OSA: apnea-hypopnea index (AHI) $\leq 5$ without hypertension, OSA: severe OSA (AHI $\geq 30$ ) without hypertension, and OSA-HTN: severe OSA with hypertension. $P$ values $<0.05$ were considered significant.

tuberous sclerosis 1 can overactivate the mTOR complex 1 pathway, thus inhibiting the differentiation of intestinal goblet cells and Paneth cells. These reductions can cause reductions in mucus and antimicrobial peptide production as well as subsequent damage to the intestinal barrier [21].

The activation of the mTOR signaling pathway is thought to be closely related to hypertension. Studies have confirmed that the protein kinase $\mathrm{B} / \mathrm{mTOR}$ pathway plays an important role in developing cerebrovascular diseases [24]. SCFAs can bind to the GPR43 as well as promote the production of regenerating islet-derived protein III- $\gamma$ and human $\beta$-defensins by activating the mTOR pathway of intestinal epithelial cells. Regenerating islet-derived protein III- $\gamma$ is a new type of antibacterial peptide, whereas $\beta$-defensins are peptides with broad-spectrum antibacterial activity; their reduction is directly involved in regulating the gut microbiota [38]. Chronic IH, combined with a high-salt diet, reportedly induces hypertension in an animal model. Lactobacillus rhamnosus can regulate the levels of intestinal metabolites and $\mathrm{CD} 4^{+} \mathrm{T}$-cell-induced inflammation through mTOR, thereby alleviating hypertension development and suggesting a correlation between the gut microbiome and 

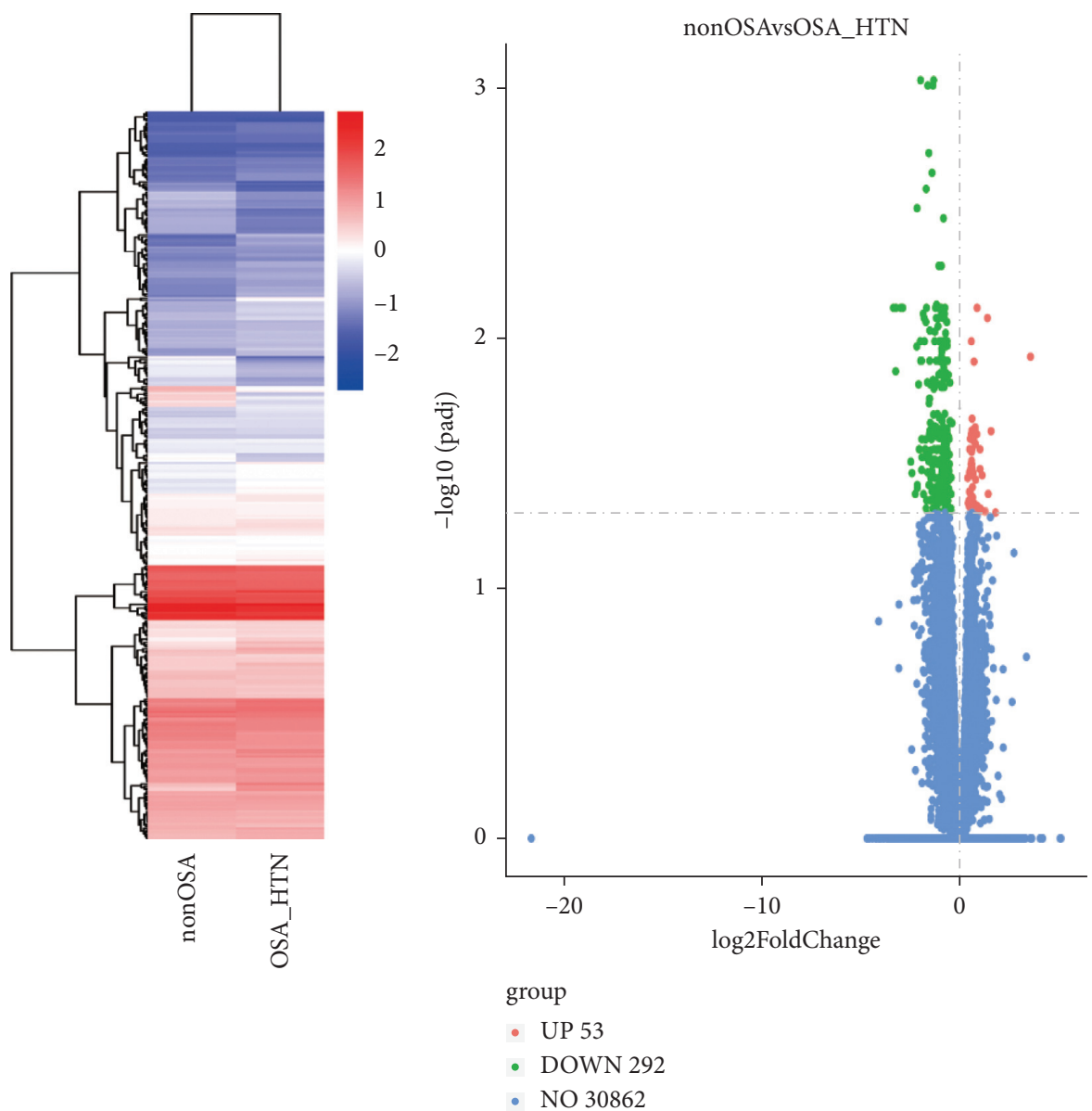

(a)

(b)

Figure 2: Continued. 


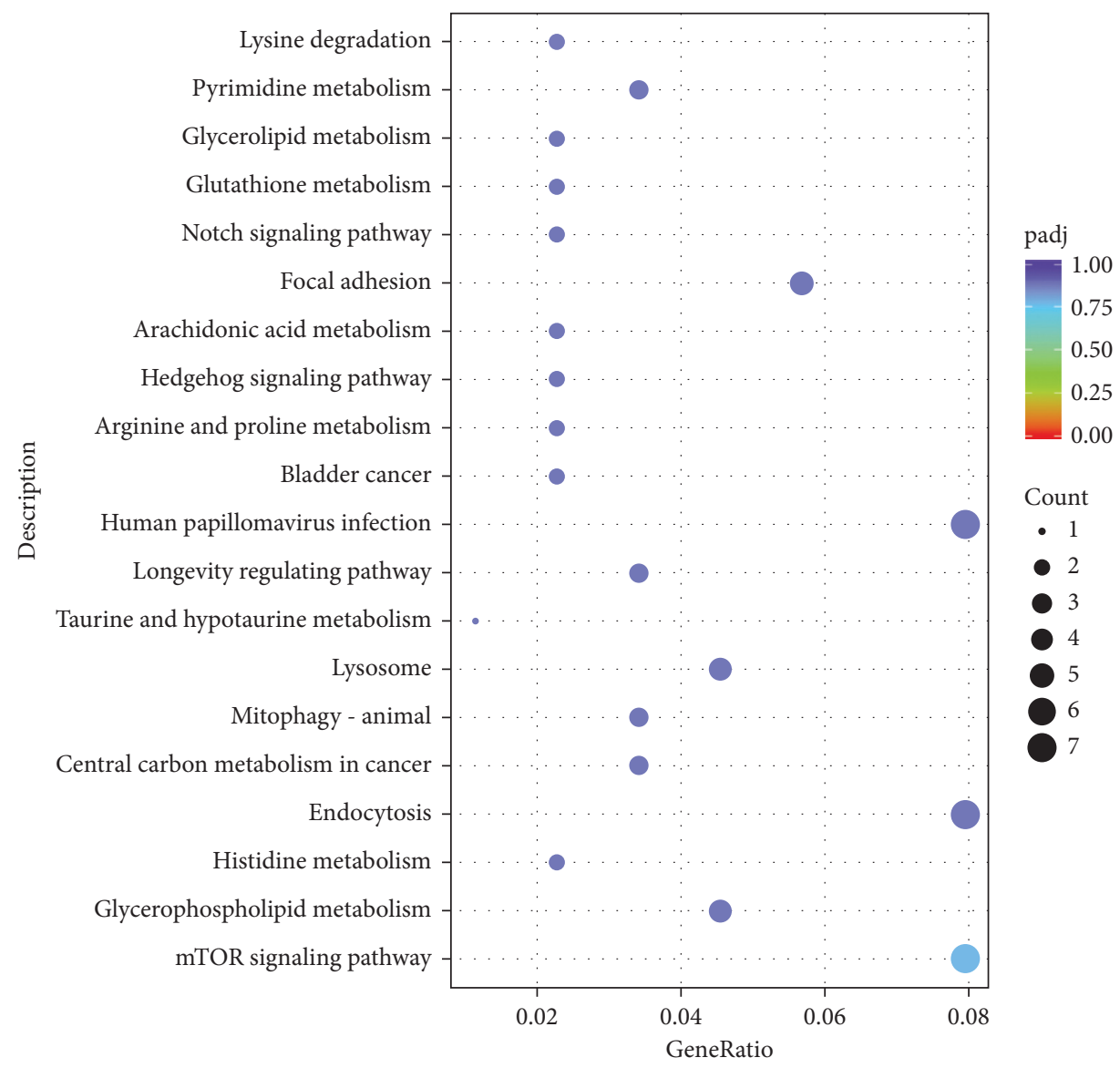

(c)

FIGURE 2: Profiles of differentially expressed gene transcripts between control patients without obstructive sleep apnea (non-OSA; apneahypopnea index (AHI) $\leq 5$ without hypertension) and patients with OSA and hypertension (OSA-HTN; AHI $\geq 30$ ). (a) Hierarchical heat maps, (b) volcano plots, and (c) top 20 overrepresented Kyoto Encyclopedia of Genes and Genomes pathways of the common differentially expressed genes. The colors (red, orange, green, light blue, and purple) represent the $Q$ value (i.e., the lowest false discovery rate), whereas the variably sized dots represent the number of genes involved in each pathway.

TABLE 3: Top 10 differentially expressed mRNAs in severe-OSA with HTN patients compared with non-OSA without hypertension subjects.

\begin{tabular}{lcccrc}
\hline & Upregulated mRNAs & & \multicolumn{2}{c}{ Downregulated mRNAs } \\
$\begin{array}{l}\text { Transcript ID } \\
\text { (Ensembl_Gene_ID) }\end{array}$ & $P$ value & $\log _{2}$ FoldChange & $\begin{array}{c}\text { Transcript ID } \\
\text { (Ensembl_Gene_ID) }\end{array}$ & $P$ value & $\log _{2}$ FoldChange \\
\hline ENSG00000123505 & $1.09 E-0.5$ & 0.871616484 & ENSG00000268350 & $8.82 E-0.8$ & -1.315999106 \\
ENSG00000242371 & $1.86 E-0.5$ & 1.397821661 & ENSG00000149591 & $1.17 E-0.7$ & -1.979312814 \\
ENSG00000079335 & $3.60 E-0.5$ & 0.585897618 & ENSG00000142552 & $1.91 E-0.7$ & -1.614114348 \\
ENSG00000196735 & $4.68 E-0.5$ & 3.57106113 & ENSG00000265982 & $2.45 E-0.7$ & -1.351438572 \\
ENSG00000102189 & $5.90 E-0.5$ & 0.722100597 & ENSG00000260428 & $5.71 E-0.7$ & -1.560721207 \\
ENSG00000087502 & 0.000142017 & 0.623605419 & ENSG00000182154 & $8.22 E-0.7$ & -1.399962275 \\
ENSG00000149308 & 0.000163471 & 0.779073878 & ENSG00000160318 & $1.11 E-0.6$ & -1.698375943 \\
ENSG00000197147 & 0.000177374 & 0.772560812 & ENSG00000265206 & $1.52 E-0.6$ & -2.155095634 \\
ENSG00000159256 & 0.000183054 & 0.612216032 & ENSG00000175482 & $1.87 E-0.6$ & -0.821303269 \\
ENSG00000134545 & 0.000188855 & 1.578550396 & ENSG00000089820 & $3.27 E-0.6$ & -0.924629667 \\
\hline
\end{tabular}

$P$ values $<0.05$ were considered significant.

mTOR immune balance in hypertension [23]. mTOR complex 1 is upregulated in PBMCs of obese patients with type 2 diabetes. It may increase the competitive inhibition of B-cell lymphoma 6 DNA binding elements by the signal transducer and activator of transcription 3 through phosphorylation of this protein, thereby weakening the inhibitory effect of B-cell lymphoma 6 on monocyte chemoattractant protein 1 , promoting the production of inflammation, and contributing to the development of atherosclerosis [25]. We previously found that the levels of 
TABLE 4: KEGG differential genes analysis in severe-OSA with HTN patients compared with non-OSA without hypertension subjects.

\begin{tabular}{lccccc}
\hline Expression & ID & Pathways & Counts & $P$ value & Gene ID \\
\hline \multirow{5}{*}{ Downregulation } & hsa04150 & mTOR signaling pathway & 6 & 0.006583727 & WDR24, DVL1, HRAS, ULK1, STK11, TELO2 \\
& hsa00564 & Glycerophospholipid metabolism & 4 & 0.015867516 & TAZ, PTDSS2, PNPLA6, DGKZ \\
& hsa05230 & Central carbon metabolism in & 3 & 0.037543817 & SIRT6, HRAS, G6PD \\
& hsa04137 & Mitophagy-animal & 3 & 0.040725158 & RHOT2, HRAS, ULK1 \\
& hsa05165 & Human papillomavirus infection & 7 & 0.052551422 & TCIRG1, SCRIB, HDAC10, RFNG, DVL1, \\
& hsa04727 & GABAergic synapse & 2 & 0.013274614 & HRAS, LAMC3 \\
\hline \multirow{5}{*}{ Upregulation } & hsa03018 & RNA degradation & 2 & 0.014053501 & SLC38A2, TRAK2 \\
& hsa05332 & Graft-versus-host disease & 1 & 0.042416288 & SKIV2L2, TTC37 \\
& hsa00340 & Histidine metabolism & 1 & 0.057882946 & CARNMT1 \\
& hsa02010 & ABC transporters & 1 & 0.100443008 & ABCD2
\end{tabular}

$P$ values $<0.05$ were considered significant.

inflammatory cytokines are elevated in OSA patients [17]. In this study, we also found that the STK11 gene was downregulated. The STK11 gene negatively regulates the mTOR signaling pathway, which can inhibit the mTOR pathway when energy is lacking. Its downregulation may result in the activation of the mTOR pathway [39]. Therefore, our findings comprehensively suggest that the regulation of the mTOR signaling pathway is involved in developing OSAassociated hypertension through its interaction with the disturbance of the gut microbiome and sleep architecture.

4.5. Limitation. First, the main limitation of this study was the small sample size, and RNA-sequencing analysis was not performed in all subjects. Second, we did not comprehensively analyze levels of SCFAs in blood or stool. Third, sleep architecture disturbances in OSA patients with or without hypertension are worthy of further investigation. Future studies should be conducted with a larger sample size and metabolites measured to address this limitation as well as carry out the microbiota transplant experiment in an animal model to examine the underlying mechanism.

\section{Conclusions}

This study revealed differences in the gut microbiome of patients with severe-OSA-HTN. Disruptions in the sleep architecture and changes in the gut microbiota may interact in OSA-associated hypertension's physiopathology, and the mTOR signaling pathway may also be involved in this critical mechanism. Our findings shed new insight into the underlying pathogenesis of OSA-associated hypertension. Further large-scale prospective cohort studies are needed to confirm these findings.

\section{Abbreviations}

OSA: $\quad$ Obstructive sleep apnea

mTOR: Mechanistic target of rapamycin

16S rRNA: $16 \mathrm{~S}$ ribosomal RNA

IH: Intermittent hypoxia

SF: $\quad$ Sleep fragmentation

CPAP: Continuous positive airway pressure
OSA-HTN: OSA and hypertension

REM: $\quad$ Rapid eye movement

NREM: Non-REM

SCFAs: Short-chain fatty acid

PSG: $\quad$ Polysomnography

PBMCs: Peripheral blood mononuclear cells

SBP: $\quad$ Systolic blood pressure

DBP: Diastolic blood pressure

KEGG: $\quad$ Kyoto Encyclopedia of Genes and Genomes

ANOVA: One-way analysis of variance

STK11: $\quad$ Serine/threonine kinase 11

D: $\quad$ Standard deviation

TNF: $\quad$ Tumor necrosis factor

IL: $\quad$ Interleukin

GPR: $\quad$ G-protein-coupled receptor

NO: $\quad$ Nitric oxide.

\section{Data Availability}

All data generated or analyzed during this study are included in this article.

\section{Ethical Approval}

All protocols were conducted and approved according to the Institutional Review Board of the Second Affiliated Hospital of Fujian Medical University (approval no. 2017-78).

\section{Conflicts of Interest}

The authors declare that they have no financial and personal relationships with others that may inappropriately influence the results and interpretation in this manuscript.

\section{Authors' Contributions}

Chih-Yuan Ko and Huan-Zhang Su have contributed equally to this work. Ko and Zeng are co-corresponding authors and have contributed equally to this work. 


\section{Acknowledgments}

The authors thank all the participants and their families who took part in this study. The authors appreciate the assistance provided by Huan Wu (G-BIO Biotech, Inc., Hangzhou, China) for performing the bioinformatic analysis. The authors would like to thank the Fujian Province Science and Technology Project, China, under contract no. 2021J01267, and the academic funding of the Second Affiliated Hospital of Fujian Medical University (serial no. BS201902).

\section{Supplementary Materials}

Supplementary Figure 1: Characteristics of sequencing data in the operational taxonomic units (OTUs) (A), the mean community diversity indices [Chao (B), Shannon (C), and Simpson (D)]. Non-OSA: apnea-hypopnea index $(\mathrm{AHI}) \leq 5$ without hypertension, OSA: severe-OSA (AHI $\geq 30)$ without hypertension, and OSA-HTN: severe OSA with hypertension. (Supplementary Materials)

\section{References}

[1] H. A. Chami, H. E. Resnick, S. F. Quan, and D. J. Gottlieb, "Association of incident cardiovascular disease with progression of sleep-disordered breathing," Circulation, vol. 123, no. 12, pp. 1280-1286, 2011.

[2] G. Parati, C. Lombardi, and K. Narkiewicz, "Sleep apnea: epidemiology, pathophysiology, and relation to cardiovascular risk," American Journal of Physiology. Regulatory, Integrative and Comparative Physiology, vol. 293, no. 4, pp. R1671-R1683, 2007.

[3] Q. Y. He, J. Feng, X. L. Zhang et al., "Sleep Breath Disorder Group; Society of Respiratory Medicine; Chinese Medical Association. Relationship of daytime blood pressure and severity of obstructive sleep apnea among Chinese: a multicenter investigation in China," Chinese Medical Journal, vol. 123, no. 1, pp. 18-22, 2010.

[4] R. P. Pedrosa, L. F. Drager, L. K. G. de Paula, A. C. S. Amaro, L. A. Bortolotto, and G. Lorenzi-Filho, "Effects of OSA treatment on BP in patients with resistant hypertension," Chest, vol. 144, no. 5, pp. 1487-1494, 2013.

[5] L. Lavie, "Oxidative stress in obstructive sleep apnea and intermittent hypoxia-revisited-the bad ugly and good: implications to the heart and brain," Sleep Medicine Reviews, vol. 20, pp. 27-45, 2015.

[6] H. Xu, H. Wang, J. Guan et al., "Effects of continuous positive airway pressure on neurocognitive architecture and function in patients with obstructive sleep apnoea: study protocol for a multicentre randomised controlled trial," BMJ Open, vol. 7, no. 5, Article ID e014932, 2017.

[7] R. K. Singh, H. W. Chang, D. Yan et al., "Influence of diet on the gut microbiome and implications for human health," Journal of Translational Medicine, vol. 15, no. 1, Article ID 73, 2017.

[8] I. Rosenzweig, S. C. Williams, and M. J. Morrell, "CrossTalk opposing view: the intermittent hypoxia attending severe obstructive sleep apnoea does not lead to alterations in brain structure and function," The Journal of Physiology, vol. 591, no. 2, pp. 383-385, 2013.

[9] O. Friedman, T. D. Bradley, P. Ruttanaumpawan, and A. G. Logan, "Independent association of drug-resistant hypertension to reduced sleep duration and efficiency,"
American Journal of Hypertension, vol. 23, no. 2, pp. 174-179, 2010.

[10] J. Jiang, Z. Gan, Y. Li et al., "REM sleep deprivation induces endothelial dysfunction and hypertension in middle-aged rats: roles of the eNOS/NO/cGMP pathway and supplementation with L-arginine," PLoS One, vol. 12, no. 8, Article ID e0182746, 2017.

[11] S. Javaheri, Y. Y. Zhao, N. M. Punjabi, S. F. Quan, D. J. Gottlieb, and S. Redline, "Slow-wave sleep is associated with incident hypertension: the sleep heart health study," Sleep, vol. 41, no. 1, Article ID zsx179, 2018.

[12] V. A. Poroyko, A. Carreras, A. Khalyfa et al., "Chronic sleep disruption alters gut microbiota, induces systemic and adipose tissue inflammation and insulin resistance in mice," Scientific Reports, vol. 6, Article ID 35405, 2016.

[13] N. Farré, R. Farré, and D. Gozal, "Sleep apnea morbidity: a consequence of microbial-immune cross-talk?" Chest, vol. 154, no. 4, pp. 754-759, 2018.

[14] I. Moreno-Indias, M. Torres, J. M. Montserrat et al., "Intermittent hypoxia alters gut microbiota diversity in a mouse model of sleep apnoea," European Respiratory Journal, vol. 45, no. 4, pp. 1055-1065, 2015.

[15] A. Tripathi, A. V. Melnik, J. Xue et al., "Intermittent hypoxia and hypercapnia, a hallmark of obstructive sleep apnea, alters the gut microbiome and metabolome," mSystems, vol. 3, no. 3, pp. e00020-18, 2018.

[16] K. M. O’Connor, E. F. Lucking, T. F. S. Bastiaanssen et al., "Prebiotic administration modulates gut microbiota and faecal short-chain fatty acid concentrations but does not prevent chronic intermittent hypoxia-induced apnoea and hypertension in adult rats," EBioMedicine, vol. 59, Article ID 102968, 2020.

[17] C.-Y. Ko, Q.-Q. Liu, H.-Z. Su et al., "Gut microbiota in obstructive sleep apnea-hypopnea syndrome: disease-related dysbiosis and metabolic comorbidities," Clinical Science, vol. 133, no. 7, pp. 905-917, 2019.

[18] C. Y. Ko, J. M. Fan, A. K. Hu et al., "Disruption of sleep architecture in Prevotella enterotype of patients with obstructive sleep apnea-hypopnea syndrome," Brain and behavior, vol. 9, no. 5, Article ID e01287, 2019.

[19] I. Robles-Vera, M. Toral, M. Romero et al., "Antihypertensive effects of probiotics," Current Hypertension Reports, vol. 19, no. 4, Article ID 26, 2017.

[20] Y. Feng, M. W. Ralls, W. Xiao, E. Miyasaka, R. S. Herman, and D. H. Teitelbaum, "Loss of enteral nutrition in a mouse model results in intestinal epithelial barrier dysfunction," Annals of the New York Academy of Sciences, vol. 1258, no. 1, pp. 71-77, 2012.

[21] Y. Zhou, P. Rychahou, Q. Wang, H. L. Weiss, and B. M. Evers, "TSC2/mTORC1 signaling controls Paneth and goblet cell differentiation in the intestinal epithelium," Cell Death \& Disease, vol. 6, no. 2, Article ID e1631, 2015.

[22] J. Li, F. Zhao, Y. Wang et al., "Gut microbiota dysbiosis contributes to the development of hypertension," Microbiome, vol. 5, no. 1, Article ID 14, 2017.

[23] J. Liu, T. Li, H. Wu et al., "Lactobacillus rhamnosus GG strain mitigated the development of obstructive sleep apnea-induced hypertension in a high salt diet via regulating TMAO level and CD4+ T cell induced-type I inflammation," Biomedicine \& Pharmacotherapy, vol. 112, Article ID 108580, 2019.

[24] X.-Y. Duan, P.-L. Xie, Y.-L. Ma, and S.-Y. Tang, "Omentin inhibits osteoblastic differentiation of calcifying vascular smooth muscle cells through the PI3K/Akt pathway," Amino Acids, vol. 41, no. 5, pp. 1223-1231, 2011. 
[25] D. Ai, H. Jiang, M. Westerterp et al., "Disruption of mammalian target of rapamycin complex 1 in macrophages decreases chemokine gene expression and atherosclerosis," Circulation Research, vol. 114, no. 10, pp. 1576-1584, 2014.

[26] M. J. Jung, J. Lee, N. R. Shin et al., "Chronic repression of mTOR complex 2 induces changes in the gut microbiota of diet-induced obese mice," Scientific Reports, vol. 6, Article ID 30887, 2016.

[27] J. C. Tudor, E. J. Davis, L. Peixoto et al., "Sleep deprivation impairs memory by attenuating mTORC1-dependent protein synthesis," Science Signaling, vol. 9, no. 425, Article ID ra41, 2016.

[28] C. Ramanathan, N. D. Kathale, D. Liu et al., "mTOR signaling regulates central and peripheral circadian clock function," PLoS Genetics, vol. 14, no. 5, Article ID e1007369, 2018.

[29] C.-Y. Ko, A.-K. Hu, D. Chou et al., "Analysis of oral microbiota in patients with obstructive sleep apnea-associated hypertension," Hypertension Research, vol. 42, no. 11, pp. 1692-1700, 2019.

[30] Y.-C. Chen, T.-W. Chen, M.-C. Su et al., "Whole genome DNA methylation analysis of obstructive sleep apnea: IL1R2,NPR2,AR,SP140Methylation and clinical phenotype," Sleep, vol. 39, no. 4, pp. 743-755, 2016.

[31] M. A. R. Vinolo, H. G. Rodrigues, R. T. Nachbar, and R. Curi, "Regulation of inflammation by short chain fatty acids," Nutrients, vol. 3, no. 10, pp. 858-876, 2011.

[32] C. Benedict, H. Vogel, W. Jonas et al., "Gut microbiota and glucometabolic alterations in response to recurrent partial sleep deprivation in normal-weight young individuals," Molecular Metabolism, vol. 5, no. 12, pp. 1175-1186, 2016.

[33] L. Galland, "The gut microbiome and the brain," Journal of Medicinal Food, vol. 17, no. 12, pp. 1261-1272, 2014.

[34] G. Clarke, S. Grenham, P. Scully et al., "The microbiome-gutbrain axis during early life regulates the hippocampal serotonergic system in a sex-dependent manner," Molecular Psychiatry, vol. 18, no. 6, pp. 666-673, 2013.

[35] I. Madaeva, O. Berdina, V. Polyakov, and S. Kolesnikov, "Obstructive sleep apnea and hypertension in adolescents: effect on neurobehavioral and cognitive functioning," $\mathrm{Ca}$ nadian Respiratory Journal, vol. 2016, Article ID 3950914, 6 pages, 2016.

[36] A. Gürün Kaya, B. Gülbay, and T. Acıcan, "Clinical and polysomnographic features of hypertension in obstructive sleep apnea: a single-center cross-sectional study," The Anatolian Journal of Cardiology, vol. 23, no. 6, pp. 334-341, 2020.

[37] J. L. Pluznick, "Microbial short-chain fatty acids and blood pressure regulation," Current Hypertension Reports, vol. 19, no. 4, Article ID 25, 2017.

[38] Y. Zhao, F. Chen, W. Wu et al., "GPR43 mediates microbiota metabolite SCFA regulation of antimicrobial peptide expression in intestinal epithelial cells via activation of mTOR and STAT3," Mucosal Immunology, vol. 11, no. 3, pp. 752-762, 2018.

[39] W. Zhou, A. I. Marcus, and P. M. Vertino, "Dysregulation of mTOR activity through LKB1 inactivation," Chinese Journal of Cancer, vol. 32, no. 8, pp. 427-433, 2013. 